\title{
I mafiosi di Leonardo Sciascia. Analisi di una riscrittura
}

\author{
Izvorni znanstveni rad \\ Original scientific paper
}

UDK 821.131.1'282-2(450.82)"19"

\begin{abstract}
Nell'ambito della produzione letteraria di Leonardo Sciascia, le opere teatrali occupano certamente un posto minore. Sono solo tre, infatti, i testi teatrali scritti da Sciascia, tutti composti e messi in scena tra il 1965 e il 1970. Ci riferiamo in particolare a L'Onorevole (1965), I mafiosi (1965) e Recitazione della controversia liparitana dedicata ad A. D. (1969), oggi raccolti in un unico volume edito da Adelphi. Scopo di questo intervento non è però tratteggiare le peculiarità del teatro dell'autore di Racalmuto, cosa che risulterebbe per altro utilissima, visto lo scarso contributo teorico e critico offerto a questa parte della produzione dell'autore siciliano. L'intento è invece porre a confronto I mafiosi di Sciascia con il testo originale, ossia I mafiusi di la Vicaria di Gaspare Mosca e Giuseppe Rizzotto del 1863. Nel prosieguo del lavoro, oltre a spiegare le differenze tra il testo originale e la versione del 1965 di Sciascia, si delinea il motivo che rende il testo sciasciano unico non solo nell'ambito della sua produzione letteraria ma anche in quello della letteratura siciliana.
\end{abstract}

Parole chiave: I mafiusi di la Vicaria, I mafiosi, teatro dialettale siciliano, letteratura siciliana, mafia, politica

$\mathrm{N}$ ell'ampia esegesi critica dell'opus sciasciano finora prodotta, il testo teatrale de I mafiosi non ha goduto della dovuta attenzione ${ }^{1}$. Nel 1966, all'indomani della prima rappresentazione di questa commedia, i giornali riportarono un breve dibattito incentrato sull'interpretazione del lavoro. Col passare degli anni, però, l'interesse verso quest'opera è venuto a mancare ${ }^{2}$. Infatti, fatta eccezione per alcuni articoli giornalistici usciti nel corso del 1968 e del 1973, la cui pubblicazione coincise con una nuova serie di rappresentazioni della commedia sciasciana, gli anni che seguirono furono caratterizzati da un notevole

\footnotetext{
Cfr. Ambroise 1974; Cattanei 1978; Di Grado 1986; Gioviale 1993; Mauro 1970; Motta 1985; Onofri 2004; Traina 1999.

2 Scritta nel 1965, I'opera venne rappresentata per la prima volta al Teatro Piccolo di Milano nel marzo del 1966. Nel corso dello stesso periodo i lettori ebbero l'occasione di leggere una serie di recensioni su questa commedia. Si vedano ad esempio Barbara 1966; Radice 1966; Terron 1966; Toschi 1966. L'opera fu pubblicata per la prima volta su "II Dramma", nn. 11 - 12, novembre - dicembre, 1972. Per quanto concerne la data della prima messa in scena ci rifacciamo al testo di Guido Nicastro (Nicastro 1978; 233) in quanto, secondo Giuseppe Traina, la commedia sarebbe stata rappresentata per la prima volta nel 1965 (Traina 1999; 144).
} 
disinteresse della critica verso questo testo ${ }^{3}$. Pertanto, al breve capitolo di Giuseppe Traina ${ }^{4}$, si possono affiancare solo due studi che fino a oggi hanno proposto un'interpretazione critica di più ampio respiro di questo lavoro: il primo è stato scritto nel 1978 da Guido Nicastro, $A$ proposito di un rifacimento: "I mafiosi" di Leonardo Sciascia, e l'altro da Erika Monforte, che ha analizzato l'opera teatrale del Nostro nella monografia I teatri di Leonardo Sciascia, edita nel $2001^{5}$. II motivo che presiede alla scelta di esaminare in questo saggio l'opera teatrale di Sciascia, oltre alla scarsità di lavori pubblicati sull'argomento, se si escludono quelli appena citati, è la convinzione che a proposito di questo testo non tutto sia stato ancora detto, e che sia pertanto possibile dare un ulteriore contributo alla sua interpretazione. Infatti, dal confronto delle analisi presentate dagli autori dei testi sopra citati, appare evidente che, pur essendo ricche di spunti interessanti e di ampie indagini sia strutturali e sia testuali, esse non chiariscono del tutto il motivo che spinse Sciascia a riprendere in mano un testo risalente al 1863. II presente saggio, pertanto, oltre a proporre un nuovo elemento sulla genesi de I mafiusi di la Vicaria ${ }^{6}$ e un raffronto con il rifacimento dell'opera proposto da Sciascia, incentrato specialmente sull'analisi dell'aspetto strutturale, cerca di capire e spiegare più a fondo la natura di questa riscrittura.

Nel periodo intercorso tra la stesura de Il giorno della civetta (edito nel 1961) e $A$ ciascuno il suo (edito nel 1966), Sciascia si era occupato anche di teatro. La commedia I mafiosi, infatti, venne composta e messa in scena nel 1966. L'opera è la riscrittura del testo teatrale I mafiusi di la Vicaria (I mafiosi della Vicaria) messo in scena per la prima volta nel 1863, nel quale si descrive la vita di un gruppo di carcerati nel penitenziario della Vicaria di Palermo ${ }^{7}$. La scelta di Sciascia non è di certo casuale se si considera che il testo è tra i più popolari del teatro dialettale siciliano. Per questo motivo le compagnie teatrali siciliane, accanto ai lavori di Alessio Di Giovanni, Nino Martoglio e Luigi Pirandello, lo mettevano spesso in scena (Barbina 1970: 5 - 11). I mafiusi di la Vicaria è un testo molto complesso sia sul piano interpretativo sia su quello della genesi. L'opera, che fu rappresentata per la prima volta sul palcoscenico palermitano del teatro Sant'Anna, pare sia stata scritta da un maestro elementare, tale Gaspare Mosca, sul finire del 1861. Sembra importante evidenziare che inizialmente l'opera era composta da soli due atti (Mosca 1895); (Loschiavo 1962: 49). Nonostante il lavoro sia oggi poco conosciuto al di fuori dei confini della Sicilia, esso è passato alla storia per l'aggettivo mafiusu contenuto nel titolo. Infatti, si deve proprio a quest'opera e al suo successo la diffusione del termine mafiusu nel significato che tutti oggi conosciamo (DELI: 702). Bisogna precisare che l'aggettivo non sarà mai ripetuto nel testo bensì in sua vece saranno usati i termini camorra e camorrista.

I due atti scritti da Mosca dunque, rappresentati in giro per la Sicilia, divennero così popolari che l'opera venne tradotta in inglese, spagnolo e italiano nonché in molti dialetti dell'Italia Settentrionale e Meridionale. Sull'onda del successo, nel 1864, pare che l'autore del testo abbia abbandonato la compagnia teatrale per l'insegnamento. II testo passò così

Cfr. Monforte 2001: 233. Si vedano poi Francica Nava 1968; Savioli 1973; Zito 1973.

$4 \quad$ Nello specifico, ci si riferisce al dizionario di Giuseppe Traina che analizza, in ordine alfabetico, tutte le opere e le tematiche legate alla produzione sciasciana. Si veda a tal proposito Traina 1999: 144 - 145.

5 V. Nicastro 1978; Monforte 2001.

$6 \quad$ Nella fattispecie si pensa a Lettera aperta del Prof. Gaspare Mosca al Sig. Alarico Lambertini direttore del periodico artistico teatrale il piccolo Faust di Bologna scritta e pubblicata nel 1895 da Gaspare Mosca, uno degli autori del testo originale. Pur non essendo stata presa in considerazione dagli autori sopra citati, questa lettera ci può dare le coordinate per esporre un'analisi quanto più attendibile delle vicende che accompagnarono la stesura di questa complessa, e per certi versi oscura, commedia teatrale del 1863.

7 Da precisare che, stando a quanto riportato da Giuseppe Guido Loschiavo, il carcere nel quale è ambientato il testo teatrale non sarebbe quello della Vicaria, che venne chiuso nel 1842, bensì quello che oggi è conosciuto come l'Ucciardone, ma che per lungo tempo è stato denominato dai palermitani Vacaria (Loschiavo 1962: 48). 
nelle mani di Giuseppe Rizzoto (Mosca 1895), capocomico e factotum dell'intera compagnia. Questi decise di allargare la commedia aggiungendo prima un terzo atto e arricchendola, in un secondo momento, anche di un prologo. L'opera, composta di quattro atti, sarà rappresentata in questa versione fino al 1894, anno della morte del Rizzotto.

I particolari inerenti l'opera e il suo destino sollecitano tutta una serie di domande alle quali tenteremo di dare una risposta. Gli studi che abbiamo consultato e le ricerche inerenti questo testo, non chiariscono il motivo che spinse il capocomico Rizzotto ad aggiungere il terzo atto e il prologo. Stando a quanto riportato in varie sedi, si ritiene che il terzo atto sia stato scritto appositamente dal Rizzotto per attenuare e per minimizzare quanto descritto nei primi due atti originali del testo teatrale (Di Bella 1991: 95); (Barbina 1970: 33 - 38). È lecito, infatti, supporre che alcuni notabili e un'ampia parte degli intellettuali palermitani, non avessero gradito il modo in cui veniva descritto e rappresentato il mondo dei carcerati del Vicaria di Palermo. Proprio per questo motivo Rizzotto venne sollecitato ad aggiungere altri atti che potessero conferire una statura morale ai personaggi descritti nella prima versione di Mosca (Di Bella 1991: 95); (Mosca 1895). Se così fosse, siamo di fronte ad un'opera che è stata manipolata e storpiata di proposito. Si deve precisare però che non tutti i critici sono dello stesso parere e che intorno alla comparsa dei due atti aggiunti dal Rizzotto esistono molte interpretazioni. Una tra tante è la teoria di Giuseppe Guido Loschiavo, secondo la quale a suggerire l'aggiunta dei due atti sarebbe stata la mafia, siccome l'opera aveva rivelato al mondo il suo gergo e la sua organizzazione (Loschiavo 1962: 49 - 50).

La consultazione di materiale documentario è stimolo per ulteriori riflessioni. La verifica delle varie e numerose riedizioni di questa commedia non consente di chiarire se si tratti di un'opera in tre o in quattro atti. Infatti, come si evince dalla consultazione di numerose ristampe, mentre alcune propongono la versione in quattro atti, altre, invece, accanto ai due atti di Mosca, aggiungono solo il terzo atto del Rizzotto, tralasciando il prologo ${ }^{8}$. Si presenta inoltre un'altra questione, relativa alla definizione dell'opera: si tratta di una commedia o di un dramma? Se Loschiavo definisce l'opera un dramma (Loschiavo 1962: 211), altre ristampe invece la definiscono una commedia (Barbina 1970: 31). Sciascia, dal canto suo, la ritiene una commedia, come risulta dall'unica prefazione a quest'opera pubblicata dall'autore (Sciascia 1995: 163). Quanto detto evidenzia la difficoltà di stabilire la genesi di quest'opera. Inoltre, nonostante molti dibattiti si siano avvicendati nel tempo, sono ancora molti i punti oscuri relativi non solo al presunto o presunti autori dell'opera, ma anche al motivo che li spinse a descrivere la mafia.

I particolari fin qui delineati aiutano a capire l'importanza della riscrittura sciasciana. Di seguito spiegheremo come l'autore di Racalmuto ha schematizzato e riorganizzato il suo testo. Quella di Sciascia si presenta come una commedia divisa in due parti senza atti e senza scene. Ciò però non deve confondere perché gli atti della commedia originale sono ben visibili all'interno del testo. Sciascia prende per buona la commedia completa, ovvero quella composta di quattro atti i quali, nella sua versione, sono così suddivisi: il prologo ed i primi due atti della commedia originale sono compressi e costituiscono quella che Sciascia delinea come prima parte, mentre il terzo atto, che è stato praticamente riscritto, occupa tutta la seconda parte dell'opera. Se nella versione sciasciana la parte strutturale è cambiata di molto rispetto all'originale, i personaggi sono rimasti gli stessi, salvo qualche piccolo taglio. Nella versione originale, infatti, troviamo i seguenti personaggi: Gioacchino Funciazza capo camorrista, Minicu Chiantedda camorrista proprietario e appartenente alla

Mentre la versione curata da Barbina in Teatro verista siciliano consta di tutti e quattro gli atti, le edizioni di Saverio Di Bella in Risorgimento e mafia in Sicilia e di Giuseppe Guido Loschiavo in 100 anni di mafia hanno solo tre atti. 
scala gerarchica più alta, Errico (o Ricu) Balata allievo nonché aspirante camorrista, Turriddu (o Turi) camorrista e maestro di Errico, Totò camorrista, Don Leonardo carcerato comune, Don Nunzio spia della polizia, Incognito (Francesco Crispi?'), Mastro Pasquale Ardichella, aiutante calzolaio, Marcello, Carceriere, Carmela, Raffaella, Graziella e Paliddu. Sciascia, dal canto suo, sfoltisce e snellisce la lunga lista di personaggi togliendo il Don Marcello del prologo, quasi tutte le figure femminili, salvo Carmela, la moglie di Gioacchino, e toglie anche il figlio di Gioacchino, Paliddu, che sarà solo nominato nella seconda parte. Ma la più evidente e significativa differenza tra le due opere è rappresentata dallo spostamento temporale attuato da Sciascia. Se I mafiusi di Mosca e Rizzotto si collocano nel 1854, nel testo di Sciascia invece le vicende iniziano esattamente il 3 aprile 1860, come si evince dal seguente passo: «Lo sanno tutti: domani, 4 aprile 1860, a Palermo e in tutto il regno di Sicilia ci sarà la rivoluzione» (Sciascia 1995: 169). Siamo dunque nei mesi che precedono lo sbarco di Garibaldi in Sicilia. Questo spostamento temporale, non privo di significato, non è casuale. Lo scopo primario, infatti, è introdurre subito la dimensione politica del fenomeno mafioso e chiarire, fin dalle prime pagine, che c'è una stretta connessione tra mafia e politica. Infatti, accennando alla rivoluzione in un testo che parla di mafiosi, Sciascia vuole mettere in risalto l'aiuto e l'apporto mafioso alla causa unitaria. Come riportano più fonti, i mafiosi si erano difatti inseriti nel complesso processo politico italiano non tanto per motivi patriottici (anche se alcuni sono ancora oggi considerati degli eroi della rivoluzione) ma piuttosto perché vedevano nell'appoggio dato a Garibaldi la possibilità di soverchiare l'ordine costituito e di creare così le condizioni ideali per poter sviluppare e allargare i propri interessi e le proprie aspirazioni ${ }^{10}$ (Marino 2008: 31 - 36); (Lupo 2004: 63 - 64). La realizzazione dei loro propositi non sarà facile, vista la tenacia di Garibaldi, a conquista ultimata, di spartire le terre ai contadini.

Oltre allo spostamento temporale, nella versione sciasciana il prologo presenta delle ulteriori novità. Nel prologo originale, che aveva ben nove scene, era rappresentata una baruffa che vedeva coinvolte le comari e la moglie di Gioacchino dove, a regnare sovrano, era il caos. Lo scopo era spiegare perché Gioacchino Funciazza fosse finito in carcere ossia il motivo che lo aveva spinto ad accoltellare a morte Gennariello, reo di aver corteggiato sua moglie Carmela. II prologo era impostato in modo che le scene fossero una sequenza continua di contraddizioni, perché il motivo che ha scatenato la rabbia di Gioacchino è frutto di un'incomprensione tra comari, determinato da uno scambio di persona (Gennariello infatti faceva la corte ad un'altra donna e non a Cramela). In Sciascia il prologo è molto ridotto, ed è incentrato solo su due personaggi, l'Incognito e Pasquale, che si scambiano una decina di battute. Il protagonista indiscusso del prologo sciasciano è l'Incognito. Nell'originale a questo personaggio sono assegnate solo quattro brevissime battute nelle quali, rivolgendosi alla moglie di Gioacchino, le chiede dove si trovi il coniuge e, non trovandolo, si gira e se

9 In questo caso il punto interrogativo è d'obbligo in quanto, nonostante gran parte della critica ritenga il personaggio dell'Incognito modellato sulla figura del noto politico siciliano Francesco Crispi (Loschiavo 1962: 49: Di Bella 1991: 95), non è chiaro da dove derivi questa interpretazione. Gli studi qui citati, che pure parlano di una tradizione popolare, non spiegano però quando e dove questa sia nata e chi sia stato il primo a vedere nell'Incognito la raffigurazione di Francesco Crispi visto che l'autore originale del testo, Gaspare Mosca, non ne parla e visto che Giuseppe Rizzotto non ha lasciato delucidazioni critiche o interpretative del testo (cfr. Mosca 1895).

10 Interessante, a questo proposito, la figura del "picciotto" Salvatore Miceli, detto Turi, che non solo viene annoverato tra le fila dei ferventi garibaldini ma soprattutto è considerato un vero e proprio patriota. Se personaggi come Miceli possono essere annoverati tra gli "eroi" di quegli anni, lo si deve non solo al loro doppio gioco ma anche, come annota lo storico Marino, al fatto che "Garibaldi, a sua volta, non andò troppo per il sottile nel vaglio delle qualità morali e dei precedenti penali di quello stupendo popolo siciliano impegnatosi nella rivoluzione nazionale.» (Marino 2008: 35) 
ne va. Nel prologo de I mafiosi innanzitutto questi non si rivolge a Carmela ma a Pasquale, I'aiuto calzolaio di Gioacchino e aspirante mafioso. II prologo sciasciano ha dunque lo scopo di presentare il personaggio principale dell'intera commedia: I'Incognito. Nella versione originale l'Incognito era concepito come un Deus ex machina, che risolve tutti i dissapori tra i personaggi (ci riferiamo soprattutto al terzo atto) ed era la figura che giustificava il legame tra mafia e politica, o meglio, tra la prima mafia e la causa unitaria (Onofri 1996: 57 - 62). S'impone a questo punto una delucidazione. L'Incognito, nella versione di Mosca e Rizzotto, ha pochissime battute e quasi tutte brevissime e a dir poco ermetiche. Infatti, incontrando Gioacchino in carcere, gli annuncia che ha da dirgli qualcosa ma alla fine i due non si parlano, lasciando così agli spettatori (o ai critici) di capire e di interpretare liberamente il loro "non parlarsi". Vista la funzione secondaria che il personaggio ha nell'originale, come ricorda anche Saverio Di Bella, molte compagnie teatrali decisero di non mettere in scena il personaggio dell'Incognito perché poco sviluppato (Di Bella 1991: 95). Ciò non ha però impedito agli studiosi, come Massimo Onofri, ad esempio, di asserire che, grazie alla figura dell'Incognito, i mafiusi sarebbero: "Un'associazione, in cui v'è molto di benigno e positivo, della quale è stato lecito servirsi in tempi di oppressione, ma che bisogna tenere sotto controllo una volta I'Unità ha avuto luogo» (Onofri 1996: 61). L'autore si riferisce soprattutto al terzo atto e al momento in cui I'Incognito minaccia di arrestare i mafiosi che, a unità ultimata, vogliono perseguire i loro scopi criminosi. Egli appare così una figura contraddittoria che, se da una parte deve spingere i carcerati - mafiosi a dare una mano alla causa unitaria, dall'altra invece deve arginare l'allargamento della loro organizzazione e della loro attività a unificazione compiuta.

In Sciascia l'Incognito, come appare evidente soprattutto nella seconda parte, subisce una drastica rivisitazione. Ne è conferma il primo atto. L'Incognito presentato da Sciascia è un personaggio certamente oscuro, ma che ha una sua personalità e dimostra, attraverso il dialogo con Pasquale, di conoscere molto bene il gergo e le regole mafiose. Riportiamo dalla prima pagina: «INCOGNITO: Siete curioso, amico mio. E se veramente voi e Gioacchino foste così (ripete il gesto di Pasquale), sapreste bene che la curiosità è un vizio pericoloso» (Sciascia 1995: 168). Il personaggio sciasciano non ha nulla da spartire con quello della versione originale che, come si è sottolineato, non trovando Gioacchino, scappa via.

Come è stato ribadito, Sciascia comprime nella prima parte dell'opera il primo e il secondo atto della commedia originale di Gaspare Mosca. Dalla piazza del quartiere dell'Albergheria a Palermo, dove era ambientato sia il prologo originale sia il dialogo tra I'Incognito e Pasquale, si passa subito al carcere della Vicaria. Nei primi due atti della versione originale si descrivevano due giorni di un gruppo sparuto di carcerati all'interno del carcere borbonico della Vicaria. La commedia, scritta da Mosca, aveva il preciso compito di spiegare al pubblico delle piazze e dei teatri quanto accadeva all'interno del carcere. A tale scopo vengono descritti tutti i rituali e le regole che si praticavano, come la riscossione del pizzo ai nuovi arrivati per la lampa della Madonna. Inoltre, si delinea la gerarchia carceraria che regolava i rapporti tra i mafiosi. Gioacchino Funciazza (nell'originale lachinu) è il capo della cosca mentre gli altri personaggi, salvo Minicu che si scoprirà essere un personaggio molto importante all'interno della società, sono per lo più dei giovani aspiranti mafiosi. Il compito di animare la storia e spiegare al pubblico il gergo malavitoso è affidato a Don Leonardo. Pur non facendo parte della società, Don Leonardo è stato preso sotto l'ala protettrice di Gioacchino il quale deve spiegargli ripetutamente il significato delle parole, dei gesti e dei comportamenti. Questa particolare funzione di uno dei personaggi permetteva di avere una sorta di tramite tra il mondo malavitoso siciliano e lo spettatore (o lettore) senza il quale il linguaggio mafioso, i loro particolari usi e la loro organizzazione potevano risultare 
incomprensibili. Nella versione di Mosca dunque la storia ruota intorno a Don Leonardo e alle sue domande. Grazie a questo stratagemma, l'autore inserisce battute nelle quali sono presenti giochi di parole che conferiscono al lavoro un carattere comico. I "dolori reumatici" diventano così "dolori romantici" oppure la "spina dorsale" diventa "spina universale". Non manca poi la figura del traditore, che in questo caso è Nunzio il quale, pur cambiando ala del carcere, viene raggiunto dalla vendetta di Gioacchino. Ne I mafiusi dunque, nonostante la breve e quasi impalpabile presenza dell'Incognito, si descriveva una sorta di "scuola" del malaffare e della delinquenza palermitana. Una "scuola" che aveva il compito di indottrinare e preparare le future forze che avrebbero certamente ingrossato le fila della mafia. Sciascia segue quasi alla lettera lo schema dei due atti originali. Ciononostante, non si può dire che le due versioni combacino in tutti i dettagli. Oltre ad una poesia popolare aggiunta da Sciascia ${ }^{11}$, la novità più importante apportata dall'autore alla sua versione è l'introduzione dell'elemento politico. In particolare ci riferiamo all'aggiunta, da parte di Sciascia, di alcuni discorsi e di alcune battute che nell'originale non figurano. In questo modo Gioacchino, già personaggio di spiccato carisma, viene tratteggiato prendendo a modello la figura del Don Mariano Arena de I/ giorno della civetta, e diventa così una guida morale e spirituale degli altri carcerati. A questo proposito è molto interessante il discorso che Gioacchino fa a Don Leonardo, che di seguito riportiamo, nel quale spiega il concetto di "umano".

GIOACCHINO: Vediamo se ci arrivo da me... Se umano vuol dire cosa dell'uomo, degli uomini, io vi dico che non c'è niente di più umano che uno tolga all'altro il pane e la camicia... E poi, caro don Leonardo, dovete tenere presente questo: il carcere è una scuola, e questi ragazzi debbono uscire di qui indottrinati a dovere... [...] Questo qui, vedete, vi taglia le falde del vestito perché sa portare rispetto solo a quelli che gli fanno paura: non ha capito che bisogna anche rispettare le persone istruite, quelle che sanno giocare con le parole, perché è con le parole che si mandano gli uomini alla forca... E non ha capito che bisogna giocare sul pane, sulla camicia e sulla pelle degli altri... (Sciascia 1995: 180).

È questo un passo denso di significati. Gioacchino, infatti, sintetizza al meglio la natura e l'atteggiamento che l'appena costituita organizzazione mafiosa deve avere nei confronti delle persone che le stanno intorno. Secondo Gioacchino, essa non deve fare la guerra a tutti bensì, se vuole sopravvivere, deve tenere strette a sé anche le persone istruite, che non sono solo l'uomo di legge ma anche l'uomo politico e, in generale, coloro che, dall'alto della loro formazione e preparazione intellettuale, vantano un ampio consenso tra la gente: la mafia insomma deve congiungersi al potere in tutte le sue forme e soprattutto senza considerare il colore del partito. È questo uno dei suggerimenti più interessanti che Sciascia mette in bocca ai suoi personaggi e che viene più volte ribadito dall'autore nel prosieguo della commedia. Indicativo il dialogo tra Gioacchino e l'Incognito, in cui il primo spiega cosa sia per lui il comando, rispondendo alla domanda postagli dall'Incognito: «E come fate a mettere d'accordo l'amore alla rivoluzione con l'ammirazione per Satriano che è venuto a reprimerla?» (Sciascia 1995: 210):

lo non metto d'accordo niente, eccellenza; non ammiro nessuno; non mi interessa né chi vince né chi perde. Quello che mi piace, della rivoluzione, sono

11 Il testo della lirica è il seguente: Carcere, vita mia, casa felice, / lo starci dentro quanto mi piace! / Testa tagliata a chi male ne dice, / a chi dice che qui non c'è la pace!/Qui solo trovi i fratelli e gli amici, / denaro, buon mangiare e allegra pace (Sciascia 1995: 170). 
le porte aperte, gli sbirri che vanno a inconigliarsi, il movimento del «levati tu che mi metto io»... Se poi la rivoluzione non capisce che il comando è comando... (Sciascia 1995: 210)

Siamo dunque di fronte al motto "fatta l'Italia facciamoci gli affari nostri" di De Roberto o, meglio ancora, siamo di fronte a quella mafia che, come i nobili de /l Gattopardo, farà di tutto affinché tutto rimanga come è. Una mafia che è pronta ad adattarsi anche a un nuovo corso politico, pur di sviluppare e allargare i propri interessi. Sciascia definisce chiaramente le connotazioni di una mafia parassitaria e approfittatrice, disposta a tutto pur di farsi largo nella nuova divisione del potere allo scopo di saziare i propri secolari appetiti di roba, fino ad allora repressi. Quella proposta da Sciascia è una visione della mafia molto esplicita, espressa senza far ricorso a discorsi ermetici e che si discosta ampiamente da quanto l'autore aveva scritto fino a quel momento. Infatti, se si prende in considerazione // giorno della civetta, si noterà come la mafia venga svelata ai lettori attraverso l'annientamento psicologico e sociale dei personaggi. I passeggeri dell'autobus che assistono all'assassinio di Salvatore Colasberna o il panellaro che, a dir poco straniato, dice di non essersi nemmeno accorto che qualcuno aveva sparato, testimoniano l'effetto che la mafia ha sulla popolazione. Nella sua versione de I mafiosi, Sciascia, pur mantenendo gran parte delle battute della versione originale della commedia, ne stravolge l'intero significato delineando una mafia cosciente dei propri scopi e del proprio ruolo nella storia. Ma l'immagine della mafia che scaturisce dalla prima parte dell'opera è anche quella di un'organizzazione spietata. Proprio per questo motivo, Sciascia attua un altro drastico cambiamento rispetto all'originale. Nella versione di Gaspare Mosca, la spia, Nunzio, viene trasferita in un'altra ala del carcere dove verrà uccisa su ordine di Gioacchino. Questo particolare, ossia l'assassinio di Nunzio, benché presumibile, non viene reso esplicito da Mosca in quanto per scrivere la lettera, che sarà poi la condanna a morte di Nunzio, l'autore si serve di tutta una serie di incomprensioni linguistiche che daranno vita a delle situazioni esilaranti, tutt'altro che drammatiche ${ }^{12}$. Sciascia invece fa morire Nunzio per chiaro ordine di Gioacchino e soprattutto lo fa uccidere nello stesso carcere, facendolo "accidentalmente" cadere dal tetto.

$\mathrm{Ci}$ occuperemo di seguito della seconda parte del testo sciasciano. Tecnicamente parlando, si tratta del terzo atto della versione originale scritto, come si è evidenziato, non da Mosca ma dal capocomico Giuseppe Rizzotto. Nel testo originale, ambientato dopo I'unificazione (lo conferma il fatto che i personaggi non parlano più di "tarì" o di "onze" ma di lire), troviamo un Gioacchino Funciazza che da capo camorrista è diventato cittadino esemplare, che vive onestamente facendo il calzolaio. L'intero atto è quasi privo di una vera e propria storia ed è essenzialmente costituito da un lungo dialogo tra gli ex carcerati e Gioacchino. I primi tentano in tutte le maniere di far rientrare nella malavita il loro ormai ex

12 Per chiarire quanto affermato, si propone il seguente passo tratto dal secondo atto, scena ottava. Don Leonardo sta scrivendo una lettera a Gioacchino, ignorando però che proprio quello che scrive sarà la condanna a morte del traditore Nunzio.

«Gioacchino In vece di: vi prego farmi il favore: vi comando e così voglio!

Leonardo Vi comando e così voglio.

Gioacchino E punto interrogatorio.

Leonardo Interrogativo volete dire? Ed a che proposito?

Gioacchino II proposito lo so io... scriva! E tre virgole.

Leonardo Non ci vanno!

Gioacchino Quattro virgole!

Leonardo Non ci vanno!

Gioacchino Cinque virgole!

Leonardo (arrabbiandosi) Dieci virgole! (eseguisce)» (Barbina 1870: 112). 
capo. Il dialogo, che quasi degenera in una mischia generale, viene interrotto dall'Incognito che informa Gioacchino della sua ammissione nella società operaia di mutuo soccorso, e minaccia di arrestare gli altri. Il terzo atto è stato completamente riscritto da Sciascia. Si può dire che il vero fulcro dell'intera commedia siasciana sia proprio questa seconda parte. Essa è costruita in modo da presentare il passaggio della mafia da fenomeno primitivo e quasi embrionale del periodo antecedente l'unità italiana, a un'organizzazione vera e propria, una volta che l'Italia "sarà fatta". Designiamo con il termine di "vera mafia" quel fenomeno, certamente settario, che aveva come unico scopo entrare in politica per controllare, attraverso gli uomini di stato, l'economia e la società siciliane.

È opportuno fare riferimento al testo sciasciano per comprendere meglio quanto appena ribadito. Siamo il giorno delle prime elezioni libere in quello che ora è il Regno d'Italia. La capitale è Roma. A essere eletti sono i neo parlamentari che andranno a costituire la camera dei deputati nella nuova capitale. Senza perdersi in discorsi inutili, Sciascia fa capire fin da subito che Gioacchino, contrariamente alla versione originale, è ancora il capo della "società" e che ora sta facendo di tutto per far eleggere l'Incognito al Parlamento. Esemplificative le prime battute tra Gioacchino e Ricu:

RICU: Dunque: per tutta la notte li ho fatti mangiare e bere a volontà, poi, li ho messi in fila, a tre a tre, i più ubriachi in mezzo, tenuti a braccetto da quelli che lo erano di meno; [...] Quando ha aperto le votazioni, il presidente, che è del partito di sua eccellenza, vedendomi con tutta quella truppa si è rallegrato «Dal mattino si vede il buon giorno», mi ha detto (Sciascia 1995: 221).

Nei primi anni del Regno, com'è risaputo, non esistevano ancora le elezioni a suffragio universale, e poche erano inoltre le persone aventi diritto al voto. Ecco dunque che la mafia si era attivata per raccogliere il maggior numero di voti destinandoli a "Sua eccellenza", I'Incognito. Per rendere la scena ancor più tragica o comica (a seconda delle interpretazioni e dei punti di vista), Sciascia apparecchia il seguente dialogo:

RICU: Il popolo sovrano!

GIOACCHINO: E che c'è da ridere?

RICU: [...] E ci sarà questa farsa ogni quattro anni?

GIOACCHINO: Non è una farsa, e tu ancora devi imparare a parlare. Il popolo ora è sovrano, proprio così. [...] E sua eccellenza, per andare a Roma a governare, ha bisogno di noi, siamo noi che lo mandiamo (Sciascia 1995: 222).

E poi di seguito:

GIOACCHINO: [...] Soddisfazioni come quella di oggi, prima di Garibaldi tu non te le saresti nemmeno sognate.

RICU: E che mangiamo, soddisfazioni?

GIOACCHINO: Perché, forse che ti manca il pane?

RICU: Il pane no; e per la verità nemmeno il formaggio.

GIOACCHINO: E nemmeno il sigaro.

RICU: E nemmeno il sigaro... Ma c'è qualcosa che non mi persuade... Non dico per me, dico per la gente... La rivoluzione, insomma... Forse non mi so spiegare. GIOACCHINO: No, non ti sai spiegare.

RICU: Mi pare, ecco, che sia andata a finire come dice il proverbio: una salma di frumento seminò, una salma raccolse (Sciascia 1995: 222 - 223). 
Pur nella sua semplicità ed elementarità questo dialogo è esemplare per intendere la storia italiana passata, ma anche quella attuale. Sciascia, infatti, ci presenta quello che già De Robeto ne I Vicerè, Pirandello ne I vecchi e i giovani e Tomasi di Lampedusa ne II gattopardo hanno spiegato e ribadito: la caduta di tutti quegli ideali che avevano spinto la gente ad unirsi ai moti unitari nel nome di una Italia finalmente unita e giusta, e la successiva delusione per la loro mancata attuazione. Quello che viene presentato è in verità il resoconto di una sconfitta perché ognuno, in questo caso la mafia, lavora non per il bene comune ma per il proprio tornaconto. Un male che affligge l'Italia appena nata e che purtroppo non è stato ancora debellato. Siamo inoltre di fronte ad una mafia rigenerata e resa più forte dalla corruttibilità degli uomini di Stato e dalla mancanza stessa dello Stato sul territorio, come ribadisce di nuovo Gioacchino nel prosieguo della commedia.

GIOACCHINO: [...] La rivoluzione, mettetevelo in testa, c'è stata; e noi siamo qui, Iontani dalla Vicaria e dalla fame, stimati da quelli che stanno in alto e rispettati da tutti gli altri, sbirri compresi, perché c’è stata... E vi voglio fare una domanda: prima un uomo come sua eccellenza quand'è che poteva avere bisogno di noi? (Sciascia 1995: 231).

È però nell'ultimo dialogo della commedia, che di seguito riportiamo, che Sciascia elabora la vera natura della mafia:

INCOGNITO: [...] Mi ha chiamato, costui, mafioso; e va dicendo che io ho portato la battaglia elettorale sul terreno della mafia... Ma qual è, o amici miei, l'autentico significato della parola mafia? Mafia è per me, per voi, eleganza, fierezza, cavalleria, senso dell'onore, superiorità, perfezione... E se mafia è questa, così come l'intendiamo noi, così come l'intende il buon popolo siciliano...

VOCE: E se è quell'altra?

Ma si vede la persona che ha lanciato la domanda crollare, colpita, e subito trascinata fuori.

INCOGNITO: Se mafia è questa, e non società per delinquere [...] ebbene, amici miei, io vi dico che sono mafioso e sarò fiero di poter portare nel Parlamento dell'Italia unita, libera, grande il soffio vivificante della mafia di questa nostra isola gloriosa... (Sciascia 1995: $242-243$ ).

È d'obbligo evidenziare che il discorso non è solo frutto della fervida immaginazione di Sciascia bensì è un discorso che gli italiani hanno davvero avuto modo di sentire. $\mathrm{Ci}$ si riferisce, infatti, alle parole del deputato nonché capo del governo italiano Vittorio Emanuele Orlando, che in più di un'occasione dichiarò: "Mafioso mi dichiaro e sono lieto di esserlo» (Lupo 2004: 207).

Da quanto esposto, appare evidente che lo scopo della riscrittura di Sciascia non è solo ripresentare, dopo cento anni, una delle commedie (o drammi) più popolari della lunga tradizione dialettale siciliana. Lo scopo dell'autore è un altro. Presentando il passaggio della mafia da una condizione quasi di primitivismo a una di assoluto potere e controllo territoriale, Sciascia punta il dito contro le istituzioni che, pur dicendo di combatterla, la mafia l'hanno spesso e volentieri assecondata. Ancora qualche riflessione finale per chiarire la natura di questa riscrittura. I mafiusi di Mosca e Rizzotto erano, come abbiamo asserito, un'opera alterata e mutata rispetto alle intenzioni originali. Portando sulle scene la sua versione, 
Sciascia volle essenzialmente cancellare questa sorta di "onta", presentando l'opera come presumibilmente doveva essere. I primi due atti di Mosca, pur descrivendo una "scuola" di mafia all'interno del carcere e pur presentando il suo particolare gergo, avevano dimostrato tutta la pericolosità di questo fenomeno. Nelle numerose scene comiche, Mosca aveva delineato difatti le caratteristiche di un'organizzazione vera e propria, con capi e sottocapi, apprendisti e semplici aspiranti, la cui forza non era nella brutalità o nel comportamento primitivo degli adepti, ma nella loro temibile e terribile intelligenza. Una visione questa che, nel corso degli anni, sarà modificata non solo dal terzo atto e dal prologo di Rizzotto, ma anche da un'idea ben diversa della mafia, che si era diffusa nel tempo. Alludiamo a quell'idea che tanta letteratura e pubblicistica avevano propagato, con il solo intento di ridimensionare il concetto di mafia. Ci riferiamo in primo luogo alle teorie del celebre demopsicologo Giuseppe Pitrè, secondo il quale la mafia era solo un atteggiamento, proprio dei giovani e dei contadini, di sfida verso le istituzioni (Pitrè 1889: 287 - 290). E ci riferiamo anche al fortunato pamphlet di Giuseppe Alongi, La maffia nei suoi fattori e nelle sue manifestazioni, dove i mafiosi sono descritti solo come un gruppo di primitivi incapaci, a suo dire, di formulare un pensiero intelligente (Alongi 1887: 51 - 82). Infine, e opportuno citare ancora l'opera teatrale di Luigi Capuana Lu Cavaleri Pidagna all'interno della quale il mafioso è presentato come un essere stupido e incapace di prendere delle decisioni sensate, che si fa comandare a bacchetta da chiunque gli paghi un piatto di maccheroni (Capuana 1912: $135)^{13}$. Così facendo, ovvero minimizzando la reale dimensione del fenomeno, si voleva dimostrare ai siciliani e al mondo intero la sua "innocenza", coprendo di ridicolo i mafiosi e mettendo in rilievo la loro incapacità di dar vita a un'organizzazione tanto complessa. Queste interpretazioni hanno impedito non solo una chiara comprensione della mafia e della complessità del fenomeno mafioso, ma hanno mostrato anche l'incapacità (volontaria o meno) della letteratura (dei letterati, in gran parte siciliani) di carpire quanto accadeva. Mettendo in scena questa riscrittura, Sciascia ha cambiato radicalmente le cose, assumendo con coraggio un atteggiamento critico nei confronti di alcuni aspetti negativi della società siciliana. Siamo dunque di fronte ad un'esplicita denuncia non solo della mafia ma anche del silenzio e della volontà di anestetizzare questo fenomeno da parte di un gruppo di intellettuali. Se con /l giorno della civetta Sciascia ha dato il via alla letteratura d'impegno, con questa riscrittura ha inteso accantonare un secolo di bugie e di falsità e di mancata presa di coscienza della realtà siciliana da parte di molti figli illustri dell'isola.

13 Riguardo a Capuana, è d'obbligo precisare che l'attacco più aspro contro coloro che vedevano nella mafia una pericolosa organizzazione lo si trova nell'ormai popolare libro - sfogo La Sicilia e il brigantaggio dove, tra le altre cose, si può leggere: "[ ] ma il daltonismo morale che sovrappone il suo falso colore a quello della realtà, ma la calunniatrice fantasia melodrammatica che ha inventato questa mafia e un brigantaggio di maniera, non avranno mai fine, non spariranno mai?» (Capuana 2005: 67). 


\section{Abbreviazioni dei dizionari citati}

DELI - Manlio Cortellazzo - Paolo Zolli, Dizionario etimologico della lingua italiana, Zanichelli, Bologna, 2009, volume unico

\section{RIFERIMENTI BIBLIOGRAFICI}

\section{ALONGI 1887}

Giuseppe Alongi, La maffia nei suoi fattori e nelle sue manifestazioni, Fratelli Bocca, Roma Torino - Firenze 1887.

\section{AMBROISE 1974}

Claude Ambroise, Invito alla lettura di Sciascia, Mursia, Milano 1974.

\section{BARBARA 1966}

Luigi Barbara, "Il ritorno dei 'Mafiosi'”, in La Nazione, 8 marzo 1966.

\section{BARBINA 1970}

Alfredo Barbina (a cura di), Teatro verista siciliano, Cappelli, Bologna 1970.

\section{CAPUANA 1912}

Luigi Capuana, Teatro dialettale siciliano, Alberto Reber, Palermo 1912.

\section{CAPUANA 2005}

Luigi Capuana, La Sicilia e il brigantaggio, Edi. bi.si., Palermo 2005.

\section{CATTANEI 1978}

Luigi Cattanei, Leonardo Sciascia, Le Monnier, Firenze 1978.

\section{BELLA 1991}

Saverio Di Bella, Risorgimento e Mafia in Sicilia: I mafiusi della Vicaria di Palermo, Pellegrini, Cosenza 1991.

\section{GRADO 1986}

Antonio Di Grado, Leonardo Sciascia, Pungitopo, Marina di Patti 1986.

\section{FRANCICA NAVA 1968}

Orazio Francica Nava, "Grande successo a Catania de 'I mafiosi' di Sciascia", in: // Giornale di Sicilia, 2 marzo 1968.

\section{GIOVIALE 1993}

Fernando Gioviale, Sciascia, Giunti \& Lisciani, Teramo 1993.

\section{LOSCHIAVO 1962}

Giuseppe Guido Loschiavo, 100 anni di mafia, Vito Bianco, Roma 1962.

\section{LUPO 2004}

Salvatore Lupo, Storia della mafia, Donzelli, Roma 2004.

\section{MONFORTE 2001}

Erika Monforte, I teatri di Leonardo Sciascia, Salvatore Sciascia, Caltanisetta - Roma 2001.

\section{MARINO 2008}

Giuseppe Carlo Marino, Storia della mafia, Newton Compton, Roma 2008.

\section{MAURO 1970}

Walter Mauro, Leonardo Sciascia, La nuova Italia, Firenze 1970.

\section{MOSCA 1895}

Gaspare Mosca, Lettera aperta del Prof. Gaspare Mosca al Sig. Alarico Lambertini direttore del periodico artistico teatrale il piccolo Faust di Bologna, Vittorio Giliberti, Palermo 1895.

\section{MOTTA 1985}

Antonio Motta, Leonardo Sciascia: la verità, l'aspra verità, Lacaita, Manduria 1985.

\section{NICASTRO 1978}

Guido Nicastro, "A proposito di un rifacimento: 'I mafiosi' di Leonardo Sciascia", in: Teatro e società in Sicilia (1860 - 1918), Bulzoni, Roma 1978.

\section{ONOFRI 1996}

Massimo Onofri, Tutti a cena da Don Mariano, Bompiani, Milano 1996.

\section{ONOFRI 2004}

Massimo Onofri, Storia di Sciascia, Laterza, Bari 2004.

\section{PITRÈ 1889}

Giuseppe Pitrè, Usi e costumi, credenze e pregiudizi del popolo siciliano, Carlo Clausen, Palermo 1889., vol. II 


\section{RADICE 1966}

Raul Radice, "Al Piccolo Teatro in scena 'I mafiosi'”, in Corriere della sera, 8 marzo 1966.

\section{SAVIOLI 1973}

Aggeo Savioli, "La galera specchio del mondo esterno", in: L'Unità, 20 marzo 1973.

\section{SCIASCIA 1995}

Leonardo Sciascia, L'onorevole, Recitazione della controversia liparitana dedicata ad A. D., I mafiosi, Adelphi, Milano 1995.

\section{TERRON 1966}

Carlo Terron, "Un copione rovesciato", in: II Tempo, 23 marzo 1966.

\section{TOSCHI 1966}

Gastone Toschi, "I mafiosi", in: Letture, aprile 1966.

\section{TRAINA 1999}

Giuseppe Traina, Leonardo Sciascia, Mondadori, Milano 1999.

\section{ZITO 1973}

Giuseppe Zito, "'I mafiosi' di Leonardo Sciascia", in: Il Secolo d'Italia, 30 marzo 1973.

\section{RIASSUNTO}

Il presente lavoro analizza le differenze tra il testo teatrale I mafiosi di Leonardo Sciascia, del 1965, e la versione originale, del 1863, I mafiusi di la Vicaria, scritta da Gaetano Mosca e Giuseppe Rizzotto. Quest'ultima era inizialmente composta di soli due atti scritti dal maestro elementare Gaetano Mosca. Nel 1864, quando questi decise di abbandonare la compagnia teatrale, il testo passò nelle mani del capocomico Giuseppe Rizzotto che, ai due atti già esistenti, aggiunse prima un terzo atto e di seguito anche un prologo. Si ritiene plausibile che l'aggiunta di questi due atti sia stata sollecitata da alcuni politici e notabili siciliani nonché da una parte degli intellettuali isolani che cercarono così di minimizzare l'immagine negativa della mafia. Un secolo dopo Leonardo Sciascia riscrisse questa commedia la quale però si discosta molto dalla versione originale. Da un punto di vista tecnico, Sciascia ha diviso il testo in due parti senza atti e scene. La vera novità è rappresentata però dal modo in cui l'autore di Racalmuto ha presentato i mafiosi e la mafia, considerata come una vera e propria organizzazione politica, l'unica in grado di garantire a un candidato la vittoria alle elezioni. Così facendo, Sciascia ha non solo descritto il vero volto della mafia, ma ha cancellato i due atti aggiunti dal Rizzotto, che per lungo tempo hanno impedito una visione obiettiva e chiara del fenomeno.

\section{SAŽETAK}

\section{Djelo I mafiusi di la Vicaria (1863) u novoj verziji Leonarda Sciascie}

Rad analizira razlike između kazališnog teksta Leonarda Sciascie I mafiosi (Mafijaši) iz 1965. godine i izvornika I mafiusi di la Vicaria (Mafijaši iz zatvora Vicaria), autora Gasparea Mosce i Giuseppea Rizzotta iz 1863. godine. Izvorna verzija imala je samo dva čina koje je napisao Mosca. Kad je on 1964. godine napustio kazališnu skupinu za koju je pisao, predstavi je dva čina dodao Rizzotto. Dodavanje ova dva čina savjetovali su ondašnji sicilijanski političari i intelektualci, kao pokušaj slabljenja opisa mafije kakva je predstavljena u prva dva originalna čina, gdje je taj fenomen predstavljen kao opasan. Leonardo Sciascia je stotinu godina kasnije ponudio djelo koje se znatno razlikuje od izvorne verzije. $S$ tehničke strane podijelio je predstavu u dva dijela, koje ne naziva činovima (atti) već prvi i drugi dio. Novina njegove verzije jest ta da Sciascia predstavlja mafijaše kao dio sicilijanske politike, a mafiju kao organizaciju koja garantira pobjedu na izborima, dok se u izvornoj verziji o politici nije govorilo. Na taj način Sciascia nije htio dokazati samo pravu prirodu mafije, već je htio doslovno izbrisati ona dva dodana čina iz izvorne verzije, koji su onemogućili cjelovito shvaćanje tog fenomena.

Ključne riječi: Mafijaši iz zatvora Vicaria, Mafijaši, sicilijansko kazalište, sicilijanska književnost, mafija, politika 